\title{
Oleic acid-induced ANGPTL4 facilitates metastasis via up-regulation of NOX4 in human colorectal cancer
}

\section{Chih-Jie Shen ${ }^{1,2,3}$, Wen-Chang Chang ${ }^{2}$, Ben-Kuen Chen ${ }^{1,2,3}$}

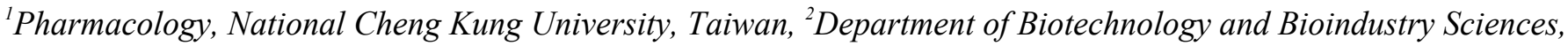
College of Bioscience and Biotechnology, National Cheng Kung University, Tainan, Taiwan, Institute for Cancer Biology and Drug Discovery, College of Medical Science and Technology, Taipei Medical University, Taipei, Taiwan

Colorectal cancer (CRC) is highly associated with metabolic diseases, such as obesity and diabetes. Elevated free fatty acids (FFAs) in cancer patients with metabolic disorders may be associated with cancer progression. In addition, increased levels of ROS are also observed in dyslipidemia which induces expression of NADPH oxidase 4 (NOX4) by FFAs in aorta, kidney, and white adipocyte in physiology condition. On the other hand, the expression of NOX4 has been also upregulated in various cancer cell types. However, the correlation between up-regulation of NOX4 and dyslipidemiaregulated CRC metastasis remains unclear. Here, we showed that oleic acid (OA) induced-angiopoietin-like 4 (ANGPTL4) expression through the activation of PPAR pathways, resulting in promoting the production of reactive oxygen species (ROS) via the induction of NOX4 expression. The knockdown of ANGPTL4 significantly inhibited OAinduced NOX4 expression. Moreover, the depletion of ROS by using NAC or knockdown of NOX4 reduced OA-induced cell invasion and extravasation. OA-induced MMP1 and MMP9 expressions were dependent on ANGPTL4 and NOX4 in CRC cells. In conclusion, OA-enhanced CRC metastasis was through the activation of ANGPTL4/NOX4/ROS/MMPs axis. Therefore, ANGPTL4 or NOX4 could be as potential therapeutic targets to improve outcome of malignant colorectal cancer. 